\title{
Methylenetetrahydrofolate reductase gene polymorphisms and cerebral palsy in Chinese infants
}

\author{
Xiuyong Cheng ${ }^{1,2,8}$, Tongchuan $\mathrm{Li}^{2,8}$, Honglian Wang ${ }^{1,5}$, Dengna $\mathrm{Zhu}^{2}$, Caiyun $\mathrm{Ma}^{3}$, Bingxiang $\mathrm{Ma}^{4}$, \\ Yinghong Wang ${ }^{4}$, Junyu Zhang ${ }^{1,5}$, Luo Guo ${ }^{1,5}$, Lei Wang ${ }^{1,5}$, Liu Yun ${ }^{1,5}$, Shiting Chen ${ }^{1,5}$, Zedong Jiang ${ }^{1,6}$, \\ Lin $\mathrm{He}^{1,5,7}$, Changlian $\mathrm{Zhu}^{2}$ and Qinghe Xing ${ }^{1,5}$
}

Genetic polymorphisms of methylenetetrahydrofolate reductase (MTHFR) have been suggested as being associated with cerebral palsy (CP) but the evidence is uncertain. The purpose of this study was to investigate whether MTHFR gene polymorphisms contribute to the development of CP in Chinese infants. For this study, 169 health controls and 159 infants with CP including 43 cases also suffering from mental retardation (MR) were recruited. Genomic DNA was prepared from venous blood and all five single nucleotide polymorphisms in MTHFR (rs4846049, rs1476413, rs1801131, rs1801133 and rs9651118) were genotyped using TaqMan technology. There were no significant differences in allele or genotype frequencies between the CP patients and controls at any of the five genetic polymorphisms. Subgroup analysis found statistically significant difference in allele and genotype frequencies between cases with both CP and MR (CP + MR) compared with both CP-only cases and controls at rs 4846049 , rs 1476413 and rs 1801131 . The frequencies of the $T$ alleles of rs 4846049 , rs 1476413 and the $G$ allele of rs 1801131 were greater in the CP + MR patients than in the CP-only patients and controls. This study provides the first evidence pointing to a MTHFR gene polymorphism as a potential risk factor for CP combined with MR.

Journal of Human Genetics (2011) 56, 17-21; doi:10.1038/jhg.2010.127; published online 21 October 2010

Keywords: cerebral palsy; gene polymorphism; mental retardation; MTHFR

\section{INTRODUCTION}

Cerebral palsy (CP) covers a group of non-progressive chronic disorders of motor function and posture caused by lesions of the developing fetal or infant brain. ${ }^{1,2}$ The characteristic signs of $\mathrm{CP}$ are spasticity, movement disorders, muscle weakness, ataxia and rigidity. The clinical classification of CP is based on motor deficit and other combined symptoms; such as mental retardation (MR), epilepsy, optic and hearing loss. ${ }^{3} \mathrm{CP}$ is the most common cause of severe physical disability in childhood, occurring in 1-2/1000 live births. ${ }^{4}$ Its prevalence rises dramatically with decreasing gestational age at birth. ${ }^{5}$ The possible risk factors for $\mathrm{CP}$ are abundant with each making only a small contribution, many cases are multifactorial in origin and exhibit marked etiologic heterogeneity. Risk factors for CP can be categorized as prenatally, perinatally and postnatally acquired of which about 70 $80 \%$ are acquired prenatally. ${ }^{6} \mathrm{~A}$ mounting body of recent evidence points to genetic influences on the occurrence of CP. This evidence includes familial data, ${ }^{7}$ twin studies ${ }^{8}$ and specific genetic factors, ${ }^{9-12}$ and indicate that $\mathrm{CP}$ may be related to genomic factors, as well as to environmental incursions during brain development.
Methylenetetrahydrofolate reductase (MTHFR) catalyses irreversibly the conversion of 5,10-methylenetetrahydrofolate to 5-methyltetrahydrofolate, which is the methyl donor for methionine synthesis from homocysteine and a key single carbon donor that participates in S-adenosylmethionine synthesis and the methylation of DNA. MTHFR also has an important role in the modulation of plasma homocysteine status by converting it into methionine. Studies showed that MTHFR gene polymorphisms are associated with inherited thrombophilias, ${ }^{13-15}$ which can result in adverse pregnancy outcomes such as CP. ${ }^{16}$ However, the evidence on association between MTHFR and CP is somewhat contradictory. ${ }^{13,17,18}$ We concluded that there was a need for a large case-control study to investigate the association of MTHFR polymorphisms and CP, particularly the Chinese context. The present study therefore focuses on the potential linkage between MTHFR polymorphisms and CP in infants in central China.

\section{SUBJECTS AND METHODS}

Subjects

The study population consisted of 159 CP patients (50 girls 31.4\%, 109 boys $68.6 \%$, mean age \pm s.d.: $16.9 \pm 14.4$ months) chosen from centers for $\mathrm{CP}$

${ }^{1}$ Department of Pediatrics, Children's Hospital of Fudan University, Shanghai, PR China; ${ }^{2}$ Department of Pediatrics, the Third Affiliated Hospital of Zhengzhou University, Zhengzhou, PR China; ${ }^{3}$ Department of Pediatrics, Zhengzhou Children's Hospital, Zhengzhou, PR China; ${ }^{4}$ Department of Pediatrics, the First Affiliated Hospital, Henan Traditional Chinese Medical College, Zhengzhou, PR China; ${ }^{5}$ Institute of Biomedical Science, Fudan University, Shanghai, PR China; ${ }^{6}$ Department of Paediatrics, University of Oxford, Oxford, UK and ${ }^{7}$ Bio-X Center, Key Laboratory for the Genetics of Developmental and Neuropsychiatric Disorders, Shanghai Jiaotong University, Shanghai, PR China

8These authors contributed equally to this work.

Correspondence: Dr C Zhu, Department of Pediatrics, the Third Affiliated Hospital of Zhengzhou University, Kangfuqian street 7, Zhengzhou 450052, PR China.

E-mail: zhuc@zzu.edu.cn or

Dr Q Xing, Institute of Biomedical Science, Fudan University, Shanghai, PR China.

E-mail: xingqinghe@hotmail.com

Received 10 May 2010; revised 26 July 2010; accepted 27 September 2010; published online 21 October 2010 
rehabilitation in the Third Affiliated Hospital of Zhengzhou University, Zhengzhou Children's Hospital and the First Affiliated Hospital of the Henan Traditional Chinese Medical College from 1 May 2008 to 31 Oct 2009. Of the total 159 cases, 43 cases also suffered from MR. The 169 healthy control subjects included in the study were chosen from the Child Healthcare Department at the same hospital during the same period and were matched for age, sex and ethnicity ( 64 girls $37.9 \%, 105$ boys $62.1 \%$, mean age \pm s.d.: $16.4 \pm 13.8$ months). All subjects including CP patients and healthy controls were Han Chinese from Henan province and had been informed consent of this study. CP patients were diagnosed by a child neurologist either by clinical examination or by using medical records. MR was identified by a specialist from the Department of Child Development based on the Bayley Scales measurement of mental developmental index $(<70) .{ }^{19}$ Children with hypotonia, ataxia, myopathy, genetic syndrome or chromosomal anomaly were excluded. Approval for the study was obtained from the ethics committee of Zhengzhou University in accordance with the Helsinki declaration.

\section{Genotyping}

A total of five single nucleotide polymorphisms (SNPs), whose minor allele frequencies in the Chinese Han population were more than 0.1, were selected from the dbSNP database (www.ncbi.nlm.nih.gov/SNP) and the hapmap human SNP database (www.hapmap.org). The five SNPs are rs4846049 in 3'UTR, rs1476413 in intron 10, rs1801131 in exon 7, rs1801133 in exon 5 and rs9651118 in intron 2, respectively. Of these, rs1801131 (A1298C) and rs1801133 (C677T) are known functional variants of MTHFR. Genomic DNA was prepared from venous blood using AxyPrep Blood Genomic DNA Miniprep Kit (Axygen Biosciences, Union city, CA, USA) according to the recommended procedure. SNPs were genotyped on the ABI 7900 DNA detection system (Applied Biosystems, Foster City, CA, USA) using TaqMan technology and the probes were designed by the Applied Biosystems service. A standard $5 \mu$ PCR reaction was carried out using TaqMan Universal PCR Master Mix reagent kits under the guidelines provided. The person who analyzed the genotype results was blind to the clinical data.

\section{Statistical analysis}

We conducted Hardy-Weinberg equilibrium tests, allele and genotype frequency analysis online on the SHESIS software platform (http://analysis. bio-x.cn/). Linkage disequilibrium was measured using standardized $\mathrm{D}^{\prime}$, and the discrepancies in allele and genotype frequencies on single loci between patients and controls were compared using a Monte Carlo simulation strategy. The program SNPSpD (http://gump.qimr.edu.au/general/daleN/matSpD/), which takes marker linkage disequilibrium information into consideration, was used to correct for multiple testing performed on each individual SNP. The numbers of observations for each haplotype were compared using $\chi^{2}$ tests. Bonferroni correction was applied for haplotype analysis. All reported $P$-values were two-tailed and statistical significance was set at $P<0.05$.

\section{RESULTS}

The frequencies of alleles/genotypes of the five investigated SNPs are listed in Table 1 . The $\chi^{2}$ goodness-of-fit test showed that the genotypic distribution of the five SNPs in both CP patients and controls was in Hardy-Weinberg equilibrium. For the totality of the subjects, there were no significant differences of allele or genotype frequencies between CP patients and controls at any of the five genetic polymorphisms. Subgroup analysis was done according to gender, gestation age, birth weight, birth asphyxia and combined CP + MR in CP patients. Significant differences in allele and genotype frequencies were observed between CP + MR patients and controls at rs4846049 $(P=0.031$ after SNPSpD correction), rs1476413 ( $P=0.028$ after SNPSpD correction) and rs1801131 $(P=0.014$ after SNPSpD correction) (Table 1), and a similar differential pattern for these three SNP allele/genotype frequencies was observed between the $\mathrm{CP}+\mathrm{MR}$ and CP-only patients (rs4846049, $P=0.016$; rs1476413, $P=0.027$; rs1801131, $P=0.004$ after SNPSpD correction) (Table 2). The frequencies of the $\mathrm{T}$ allele of rs4846049, the T allele of rs1476413 and the G allele of rs1801131 (A1298C) were greater in CP + MR patients

Table 1 Allele and genotype frequencies of SNPs in MTHFR

\begin{tabular}{|c|c|c|c|c|c|c|c|c|}
\hline \multirow{2}{*}{$\frac{\text { Group }}{\text { rs4846049 }}$} & \multicolumn{2}{|c|}{ Allele frequency } & \multirow[t]{2}{*}{$\mathrm{P}$} & \multicolumn{3}{|c|}{ Genotype frequency } & \multirow[t]{2}{*}{$P$} & \multirow[t]{2}{*}{$H-W$} \\
\hline & G & $\mathrm{T}$ & & $\mathrm{G} / \mathrm{G}$ & $\mathrm{G} / \mathrm{T}$ & $\mathrm{T} / \mathrm{T}$ & & \\
\hline $\mathrm{CP}+\mathrm{MR}$ & $65(0.756)$ & $21(0.244)$ & 0.014 & $25(0.581)$ & $15(0.349)$ & $3(0.070)$ & 0.009 & 0.719 \\
\hline $\mathrm{CP}$ & $271(0.852)$ & $47(0.148)$ & 0.668 & $115(0.723)$ & $41(0.258)$ & $3(0.019)$ & 0.565 & 0.766 \\
\hline Control & $292(0.864)$ & $46(0.136)$ & & $124(0.734)$ & $44(0.260)$ & $1(0.006)$ & & 0.163 \\
\hline rs1476413 & C & $\mathrm{T}$ & & $\mathrm{C} / \mathrm{C}$ & $\mathrm{C} / \mathrm{T}$ & $\mathrm{T} / \mathrm{T}$ & & \\
\hline $\mathrm{CP}+\mathrm{MR}$ & $65(0.756)$ & $21(0.244)$ & 0.011 & $25(0.581)$ & $15(0.349)$ & $3(0.070)$ & 0.008 & 0.719 \\
\hline $\mathrm{CP}$ & $270(0.849)$ & $48(0.151)$ & 0.513 & $114(0.717)$ & $42(0.264)$ & $3(0.019)$ & 0.545 & 0.700 \\
\hline Control & $293(0.867)$ & $45(0.133)$ & & $125(0.740)$ & $43(0.254)$ & $1(0.006)$ & & 0.183 \\
\hline rs1801131 & $\mathrm{G}$ & $\mathrm{T}$ & & $\mathrm{G} / \mathrm{G}$ & $\mathrm{G} / \mathrm{T}$ & $\mathrm{T} / \mathrm{T}$ & & \\
\hline $\mathrm{CP}+\mathrm{MR}$ & $21(0.244)$ & $65(0.756)$ & 0.004 & $3(0.070)$ & 15 (0.349) & $25(0.581)$ & 0.004 & 0.719 \\
\hline $\mathrm{CP}$ & $44(0.138)$ & $274(0.862)$ & 0.515 & $3(0.019)$ & $38(0.239)$ & $118(0.742$ & 0.549 & 0.977 \\
\hline Control & $41(0.121)$ & $297(0.879)$ & & $1(0.006)$ & $39(0.231)$ & $129(0.763)$ & & 0.283 \\
\hline rs1801133 & $A$ & $G$ & & $\mathrm{~A} / \mathrm{A}$ & $A / G$ & $\mathrm{G} / \mathrm{G}$ & & \\
\hline $\mathrm{CP}+\mathrm{MR}$ & $51(0.593)$ & $35(0.407)$ & 0.667 & $15(0.349)$ & $21(0.488)$ & $7(0.163)$ & 0.800 & 0.939 \\
\hline $\mathrm{CP}$ & $208(0.654)$ & $110(0.346)$ & 0.162 & $69(0.434)$ & $70(0.440)$ & $20(0.126)$ & 0.358 & 0.733 \\
\hline Control & $209(0.618)$ & $129(0.382)$ & & $61(0.361)$ & $87(0.515)$ & $21(0.124)$ & & 0.238 \\
\hline rs9651118 & C & $\mathrm{T}$ & & $\mathrm{C} / \mathrm{C}$ & $\mathrm{C} / \mathrm{T}$ & $\mathrm{T} / \mathrm{T}$ & & \\
\hline $\mathrm{CP}+\mathrm{MR}$ & $13(0.151)$ & $73(0.849)$ & 0.097 & $1(0.023)$ & $11(0.256)$ & $31(0.721)$ & 0.262 & 0.983 \\
\hline $\mathrm{CP}$ & $62(0.195)$ & $256(0.805)$ & 0.227 & $9(0.057)$ & $44(0.277)$ & $106(0.667)$ & 0.348 & 0.135 \\
\hline Control & $79(0.234)$ & $259(0.766)$ & & $10(0.059)$ & $59(0.349)$ & $100(0.592)$ & & 0.742 \\
\hline
\end{tabular}

Abbreviations: CP+MR, cerebral palsy combined with mental retardation; H-W, Hardy-Weinberg; MTHFR, methylenetetrahydrofolate reductase; SNP, single nucleotide polymorphism. 
Table 2 Distribution of MTHFR alleles and genotypes in CP cases with and without MR

\begin{tabular}{|c|c|c|c|c|c|c|c|c|}
\hline \multirow{2}{*}{$\frac{\text { Group }}{\text { rs4846049 }}$} & \multicolumn{2}{|c|}{ Allele frequency } & \multirow[t]{2}{*}{$\mathrm{P}$} & \multicolumn{3}{|c|}{ Genotype frequency } & \multirow[t]{2}{*}{$\mathrm{P}$} & \multirow[t]{2}{*}{$H-W$} \\
\hline & G & $\mathrm{T}$ & & $\mathrm{G} / \mathrm{G}$ & $\mathrm{G} / \mathrm{T}$ & $\mathrm{T} / \mathrm{T}$ & & \\
\hline $\mathrm{CP}+\mathrm{MR}$ & $65(0.756)$ & $21(0.244)$ & 0.005 & $25(0.581)$ & $15(0.349)$ & $3(0.070)$ & 0.006 & 0.719 \\
\hline rs1476413 & C & $\mathrm{T}$ & & $\mathrm{C} / \mathrm{C}$ & $\mathrm{C} / \mathrm{T}$ & $T / T$ & & \\
\hline $\mathrm{CP}+\mathrm{MR}$ & $65(0.756)$ & $21(0.244)$ & 0.007 & $25(0.581)$ & $15(0.349)$ & $3(0.070)$ & 0.008 & 0.719 \\
\hline rs1801131 & G & $\mathrm{T}$ & & G/G & $\mathrm{G} / \mathrm{T}$ & $\mathrm{T} / \mathrm{T}$ & & \\
\hline $\mathrm{CP}+\mathrm{MR}$ & $21(0.244)$ & $65(0.756)$ & 0.001 & $3(0.070)$ & $15(0.349)$ & $25(0.581)$ & 0.003 & 0.719 \\
\hline$C P^{*}$ & $19(0.097)$ & $177(0.903)$ & & $0(0.000)$ & $19(0.194)$ & $79(0.806)$ & & 0.288 \\
\hline rs1801133 & A & G & & $\mathrm{A} / \mathrm{A}$ & $A / G$ & $\mathrm{G} / \mathrm{G}$ & & \\
\hline $\mathrm{CP}+\mathrm{MR}$ & $13(0.151)$ & $73(0.849)$ & 0.186 & $1(0.023)$ & $11(0.256)$ & $31(0.721)$ & 0.418 & 0.983 \\
\hline$C P^{*}$ & $43(0.219)$ & $153(0.781)$ & & $7(0.071)$ & $29(0.296)$ & $62(0.633)$ & & 0.178 \\
\hline
\end{tabular}

Abbreviations: $\mathrm{CP}+\mathrm{MR}$, cerebral palsy combined with mental retardation; $\mathrm{CP}^{*}, \mathrm{CP}$ without MR; MTHFR, methylenetetrahydrofolate reductase.

Table 3 The linkage disequilibrium among the SNPs

\begin{tabular}{lccccc}
\hline$D / r^{2}$ & $r s 4846049$ & $r s 1476413$ & $r s 1801131$ & $r s 1801133$ & $r s 9651118$ \\
\hline$r s 4846049$ & & 0.962 & 0.972 & 0.862 & 0.997 \\
rs1476413 & 0.926 & & 0.972 & 0.862 & 0.997 \\
rs1801131 & 0.852 & 0.852 & & 0.975 & 0.997 \\
rs1801133 & 0.214 & 0.214 & 0.247 & & 0.960 \\
rs9651118 & 0.045 & 0.045 & 0.041 & 0.441 &
\end{tabular}

Abbreviation: SNP, single nucleotide polymorphism.

The standardized $D^{\prime}$ values are shown above the diagonal, and the $r^{2}$ values are shown below the diagonal.

than in controls or CP-only patients. No significant differences were detected in either allele or genotype frequencies of the other two SNPs, rs1801133 (C677T) and rs9651118. There was no difference in the allele or genotype frequencies in the other CP subgroups classified by gender, gestation age, birth weight and birth asphyxia (data not shown).

The estimation of linkage disequilibrium for all pairs of SNP markers showed strong linkage disequilibrium $\left(\mathrm{D}^{\prime}>0.8\right)$ (Table 3 ). We then constructed haplotypes of all SNPs and analyzed only the most common ones (those with a frequency $<0.01$ were excluded from the analysis). Haplotype analysis revealed a significant global $P$-value $(P=0.021$ after Bonferroni correction, Table 4$)$ and haplotype frequency discrepancies. The haplotype TTGGT (rs4846049, rs1476413, rs1801131, rs1801133 and rs9651118) was observed to be strongly associated with $\mathrm{CP}+\mathrm{MR}(P=0.001$, odds ratio $=2.695$, $95 \%$ confidence interval $=1.470 \sim 4.941, P=0.005$ after Bonferroni correction).

\section{DISCUSSION}

$\mathrm{CP}$ covers a range of neurological conditions that share common disorders of motor function and posture. The underlying cause of CP remains obscure in most cases and efforts to reduce the incidence of
Table 4 Estimated MTHFR haplotype frequencies

\begin{tabular}{|c|c|c|c|c|}
\hline \multirow[b]{2}{*}{ Haplotype } & \multicolumn{2}{|c|}{ Frequencies } & \multirow[b]{2}{*}{$\mathrm{P}$-value } & \multirow[b]{2}{*}{ OR $(95 \% \mathrm{Cl})$} \\
\hline & $C P$ with $M R$ & Controls & & \\
\hline G C TAT & $51.00(0.593)$ & $201.15(0.595)$ & 0.562 & $0.866(0.533 \sim 1.409)$ \\
\hline G C T G C & $13.00(0.151)$ & $69.91(0.207)$ & 0.172 & $0.639(0.335 \sim 1.220)$ \\
\hline G C T G T & $1.00(0.012)$ & $15.34(0.045)$ & 0.129 & $0.234(0.031 \sim 1.796)$ \\
\hline T T G G T & $21.00(0.244)$ & $34.33(0.102)$ & $0.005^{*}$ & $2.695(1.470 \sim 4.941)$ \\
\hline Global & & & $0.021 *$ & \\
\hline
\end{tabular}

Abbreviations: $\mathrm{Cl}$, confidence interval; $\mathrm{CP}$, cerebral palsy; MR, mental retardation; MTHFR, methylenetetrahydrofolate reductase; OR, odds ratio.

Loci chosen for haplotype analysis: rs4846049, rs1476413, rs1801131, rs1801133,

rs9651118. Haplotypes with a frequency $<0.01$ in both control \& case have been dropped.

* $P$-values after Bonferroni correction $(\times 5)$.

perinatal brain injury in term infants have failed despite the improvement in obstetric and neonatal care. The genetic influences affecting CP have been the subject of much attention in recent years. ${ }^{10-12,20,21}$ Several genes have been identified as risk factors, and have been categorized as thrombophilic, cytokine, apolipoprotein $\mathrm{E}$ and a group related to cardiovascular physiology and the functioning of the immune system. ${ }^{21}$ Among the candidate genes, several SNPs of the MTHFR genes have been investigated either in normal populations or CP patients. ${ }^{11,13,22}$ A case-control study from Australia found that MTHFR C677T was associated with an increased risk of developing any type of $\mathrm{CP}$ and was also associated with diplegia for all gestation periods. ${ }^{13}$ Other studies have produced contrary results and have failed to confirm an association between MTHFR C677T (rs1801133) and CP. ${ }^{11,23,24}$ In the present study of Chinese infants, there were no significant differences of allele or genotype frequencies between CP patients and controls at any of the five MTHFR gene polymorphisms. These contradictory findings might be owing to ethnic or racial-ethnic differences of folate in the diet or metabolism or to other characteristics of CP cases. The largest population study for geographical and 
ethnic variation of the MTHFR $677 \mathrm{C}>\mathrm{T}$ allele demonstrated ethnic and geographical variation. ${ }^{25}$ The frequency of the homozygous TT genotype is particularly high in northern China (20\%), southern Italy (26\%) and Mexico (32\%). The incidence of neural tube defect is high in Mexico and northern China, but not in southern Italy. ${ }^{22}$ The impact of such marked geographical and ethnic distribution of the MTHFR $677 \mathrm{C}>\mathrm{T}$ allele on the distribution of the disease is still unclear. There is no evidence to show that geographical and ethnic variations in the MTHFR $677 \mathrm{C}>\mathrm{T}$ allele is associated with CP.

The prevalence of severe MR is about 3.0/1000 live births and in most cases the causes are unknown. MR is also an important condition in Down's syndrome, hypothyroidism or CP. The prevalence of MR varies with the type of $\mathrm{CP}$ and increases significantly when epilepsy is present and is common in severely disabled CP patients. In this study, $27 \%$ of the cases suffered from both $\mathrm{CP}+\mathrm{MR}$, and this proportion is in line with other similar studies. ${ }^{26}$ An association between MTHFR polymorphisms and intelligence quotient in Down's syndrome patients has been reported, ${ }^{27}$ but not in MR-only patients. ${ }^{28}$ In the cohort for this study, we observed a significant difference in allele and genotype frequencies between $\mathrm{CP}+\mathrm{MR}$ patients and controls at rs4846049, rs1476413 and rs1801131 and there was a statistically significant difference in the frequencies of the three SNPs between $\mathrm{CP}+\mathrm{MR}$ and CP-only cases. Furthermore, the haplotype TTGGT (rs4846049, rs1476413, rs1801131, rs1801133 and rs9651118) was statistically associated with MR. A1298C (rs1801131), which changes a glutamate into an alanine residue, destroys an MboII recognition site and results in decreased MTHFR activity, has been associated with lower blood folate and higher homocysteine levels in some studies. These results suggest that folate metabolic gene polymorphisms are associated with $\mathrm{CP}+\mathrm{MR}$ and that the genes susceptible to CP differ somewhat according to the clinical CP subtype although such genes are not the only possible causes of this clinical syndrome.

Gender related pathophysiological features are thought to be related to $\mathrm{CP}^{29,30}$ We therefore, checked the gender differences in genetic susceptibility to $\mathrm{CP}$, but found no association between the five MTHFR gene polymorphisms and CP. Prematurity is another important contributor to CP. The overall reported incidence of $\mathrm{CP}$ is $1.2 / 1000$ live birth in term infants compared with $86 / 1000$ live birth in extremely premature infants. ${ }^{20}$ Studies have identified specific gene polymorphisms that specifically predispose to prematurity. ${ }^{11,31}$ In this study, we compared gestation age differences with genetic susceptibility to $\mathrm{CP}$, but, no difference was observed with respect to the MTHFR gene polymorphisms, which is in accordance with a previous report. ${ }^{11}$

In conclusion, our results support the conclusion that none of the five SNPs in MTHFR contribute to the occurrence of CP in Chinese infants. However, this is the first report to our knowledge to demonstrate that MTHFR genetic polymorphisms are associated with $\mathrm{CP}$ combined with MR. It adds to the existing evidence that certain gene variants may in some way contribute to CP. Additional investigations are needed to test the linkage of genetic factors with different types of CP. However, it should be emphasized that our findings are still preliminary, given the small number of subjects in each group and further replication studies with a larger number of subjects will be necessary to provide a more definitive answer.

\section{CONFLICT OF INTEREST}

The authors declare no conflict of interest.

\section{ACKNOWLEDGEMENTS}

We are grateful to all the participants in this study. This work was supported by a grant from national natural science foundation of China (No: 90919049), the 973 Program (2010CB529600, 2007CB947300), the Shanghai Municipal Commission of Science and Technology Program (09DJ1400601) and the third phase of 211 project from Ministry of Education of China.

1 Koman, L. A., Smith, B. P. \& Shilt, J. S. Cerebral palsy. Lancet 363, 1619-1631 (2004).

2 Rosenbaum, P., Paneth, N., Leviton, A., Goldstein, M., Bax, M., Damiano, D. et al. A report: the definition and classification of cerebral palsy April 2006. Dev. Med. Child Neurol. Suppl. 109, 8-14 (2007).

3 O'Shea, T. M. Diagnosis, treatment and prevention of cerebral palsy. Clin. Obstet. Gynecol. 51, 816-828 (2008).

4 Paneth, N., Hong, T. \& Korzeniewski, S. The descriptive epidemiology of cerebral palsy. Clin. Perinatol. 33, 251-267 (2006).

5 Liu, J. M., Li, S., Lin, Q. \& Li, Z. Prevalence of cerebral palsy in China. Int. J. Epidemiol. 28, 949-954 (1999).

6 Jacobsson, B. \& Hagberg, G. Antenatal risk factors for cerebral palsy. Best Pract. Res. 18, 425-436 (2004).

7 Lerer, I., Sagi, M., Meiner, V., Cohen, T., Zlotogora, J. \& Abeliovich, D. Deletion of the ANKRD15 gene at 9p24.3 causes parent-of-origin-dependent inheritance of familial cerebral palsy. Hum. Mol. Genet. 14, 3911-3920 (2005).

8 Zhang, T., Wang, M., Pan, L., Ding, W., Wang, J. G., Yang, L. et al. Study of gene expression profiles and biological mechanism of cerebral palsy using a monozygotic twin pair. Twin Res. Hum. Genet. 10, 496-507 (2007).

9 Costeff, H. Estimated frequency of genetic and nongenetic causes of congenital idiopathic cerebral palsy in west Sweden. Ann. Hum. Genet. 68, 515-520 (2004).

10 Wu, Y. W., Croen, L. A., Torres, A. R., Van De Water, J., Grether, J. K. \& Hsu, N. N. Interleukin-6 genotype and risk for cerebral palsy in term and near-term infants. Ann. Neurol. 66, 663-670 (2009).

11 Nelson, K. B., Dambrosia, J. M., Iovannisci, D. M., Cheng, S., Grether, J. K. \& Lammer, E. Genetic polymorphisms and cerebral palsy in very preterm infants. Pediatr. Res. 57, 494-499 (2005).

12 Gibson, C. S., Maclennan, A. H., Dekker, G. A., Goldwater, P. N., Sullivan, T. R., Munroe, D. J. et al. Candidate genes and cerebral palsy: a population-based study. Pediatrics. 122, 1079-1085 (2008)

13 Gibson, C. S., MacLennan, A. H., Goldwater, P. N., Haan, E. A., Priest, K. \& Dekker, G. A. The association between inherited cytokine polymorphisms and cerebral palsy. Am. J. Obstet. Gynecol. 194, 674, e1-11 (2006).

14 Moe, K. T., Woon, F. P., De Silva, D. A., Wong, P., Koh, T. H., Kingwell, B. et al. Association of acute ischemic stroke with the MTHFR C677T polymorphism but not with NOS3 gene polymorphisms in a Singapore population. Eur. J. Neurol. 15, 1309-1314 (2008).

15 Sazci, A., Ergul, E., Tuncer, N., Akpinar, G. \& Kara, I. Methylenetetrahydrofolate reductase gene polymorphisms are associated with ischemic and hemorrhagic stroke: dual effect of MTHFR polymorphisms C677T and A1298C. Brain Res. Bull. 71, 45-50 (2006).

16 Arias, F., Romero, R., Joist, H. \& Kraus, F. T. Thrombophilia: a mechanism of disease in women with adverse pregnancy outcome and thrombotic lesions in the placenta. J. Matern. Fetal. Med. 7, 277-286 (1998).

17 Smith, R. A., Skelton, M., Howard, M. \& Levene, M. Is thrombophilia a factor in the development of hemiplegic cerebral palsy? Dev. Med. Child. Neurol. 43, 724-730 (2001).

18 Peddareddygari, L. R., Dutra, A. V., Levenstien, M. A., Sen, S. \& Grewal, R. P. An analysis of methylenetetrahydrofolate reductase and glutathione S-transferase omega-1 genes as modifiers of the cerebral response to ischemia. BMC. Neurol. 9, 37 (2009).

19 Zhu, C., Kang, W., Xu, F., Cheng, X., Zhang, Z., Jia, L. et al. Erythropoietin improved neurologic outcomes in newborns with hypoxic-ischemic encephalopathy. Pediatrics. 124, e218-e226 (2009).

20 Schaefer, G. B. Genetics considerations in cerebral palsy. Semin. Pediatr. Neurol. 15, 21-26 (2008).

21 O'Callaghan, M. E., MacLennan, A. H., Haan, E. A. \& Dekker, G. The genomic basis of cerebral palsy: a HuGE systematic literature review. Hum. Genet. 126, 149-172 (2009).

22 Moore, C. A., Li, S., Li, Z., Hong, S. X., Gu, H. Q., Berry, R. J. et al. Elevated rates of severe neural tube defects in a high-prevalence area in northern China. Am. J. Med. Genet. 73, 113-118 (1997).

23 Yehezkely-Schildkraut, V., Kutai, M., Hugeirat, Y., Levin, C., Shalev, S. A., Mazor, G. et al. Thrombophilia: a risk factor for cerebral palsy? Isr. Med. Assoc. J. 7, 808-811 (2005).

24 Fattal-Valevski, A., Kenet, G., Kupferminc, M. J., Mesterman, R., Leitner, Y., Rimon, E. et al. Role of thrombophilic risk factors in children with non-stroke cerebral palsy. Thromb. Res. 116, 133-137 (2005).

25 Wilcken, B., Bamforth, F., Li, Z., Zhu, H., Ritvanen, A., Renlund, M. et al. Geographical and ethnic variation of the $677 \mathrm{C}>$ T allele of 5,10 methylenetetrahydrofolate reductase (MTHFR): findings from over 7000 newborns from 16 areas world wide. J. Med. Genet. 40, 619-625 (2003). 
26 Andersen, G. L., Irgens, L. M., Haagaas, I., Skranes, J. S., Meberg, A. E. \& Vik, T. Cerebral palsy in Norway: prevalence, subtypes and severity. Eur. J. Paediatr. Neurol. 12, 4-13 (2008).

27 Gueant, J. L., Anello, G., Bosco, P., Gueant-Rodriguez, R. M., Romano, A., Barone, C. et al. Homocysteine and related genetic polymorphisms in Down's syndrome IQ. J. Neurol. Neurosurg. Psychiatr. 76, 706-709 (2005).

28 Shaw, G. M., Jelliffe-Pawlowski, L., Nelson, V., Zhu, H., Harris, J. A. \& Finnell, R. H. Infant C677T MTHFR polymorphism and severe mental retardation. Birth Defects Res. 79, 24-26 (2007).
29 Johnston, M. V. \& Hagberg, H. Sex and the pathogenesis of cerebral palsy. Dev. Med. Child. Neurol. 49, 74-78 (2007).

30 Zhu, C., Xu, F., Wang, X., Shibata, M., Uchiyama, Y., Blomgren, K. et al. Different apoptotic mechanisms are activated in male and female brains after neonatal hypoxiaischaemia. J. Neurochem. 96, 1016-1027 (2006).

31 Gibson, C. S., MacLennan, A. H., Dekker, G. A., Goldwater, P. N., Dambrosia, J. M., Munroe, D. J. et al. Genetic polymorphisms and spontaneous preterm birth. Obstet. Gynecol. 109, 384-391 (2007). 